\title{
Right ventricular morphology and function is not related with microRNAs and fibrosis markers in dilated cardiomyopathy
}

\author{
Paweł Rubiś ${ }^{1}$, Justyna Totoń-Żurańska ${ }^{2}$, Sylwia Wiśniowska-Śmiałek ${ }^{1}$, \\ Maria Kołton-Wróż ${ }^{2}$, Paweł Wołkow ${ }^{2}$, Ewa Wypasek ${ }^{3}$, Lucyna Rudnicka-Sosin ${ }^{4}$, \\ Agnieszka Pawlak ${ }^{5,6}$, Artur Kozanecki ${ }^{1}$, Lidia Tomkiewicz-Pająk ${ }^{7}$, Piotr Podolec ${ }^{1,7}$ \\ ${ }^{1}$ Department of Cardiac and Vascular Diseases, John Paul II Hospital, Krakow, Poland \\ ${ }^{2}$ Center for Medical Genomics OMICRON, Jagiellonian University Medical College, Krakow, Poland \\ ${ }^{3}$ Department of Molecular Biology, John Paul II Hospital, Krakow, Poland \\ ${ }^{4}$ Department of Pathology, John Paul II Hospital, Krakow, Poland \\ ${ }^{5}$ Mossakowski Medical Research Centre, Polish Academy of Sciences, Warsaw, Poland \\ ${ }^{6} 2^{\text {nd }}$ Central Hospital of the Ministry of Interior, Department of Cardiology, Warsaw, Poland \\ ${ }^{7}$ Jagiellonian University, Medical Collage, Krakow, Poland
}

\begin{abstract}
Background: The relationship between right ventricle (RV), extracellular matrix (ECM) fibrosis and fibrosis-linked, circulating microRNAs in dilated cardiomyopathy (DCM) is unknown.

Aim: The aim of the study was to uncover the associations between serum markers of ECM metabolism and circulating microRNAs with RV morphological and functional parameters.

Methods: The study population consisted of 70 consecutive DCM patients (ejection fraction $24.4 \pm$ $\pm 7.4 \%)$. Based on detailed echocardiographic assessment - 15 patients had normal RV, whereas 55 patients had RV dilatation (RVD) and/or RV systolic dysfunction (RVSD). Procollagens type I and III carboxy- and amino-terminal peptides, osteopontin (OPN), TGF1- $\beta 1$, connective tissue growth factor (CTGF), MMP-2, MMP-9 and TIMP-1 were measured in serum as well as expression of miR-21, miR-26, miR-29, miR-30 and miR-133a. All patients underwent endomyocardial biopsy.

Results: Biopsy-proven fibrosis was evenly distributed in two groups. Serum levels of fibrosis markers did not differ between groups. OPN, CTGF, MMP-2, and TIMP-1 correlated with RV parameters. Only miR-133 a was differently expressed in both groups. MiR-21, miR-26, miR-30, and miR-133a correlated with $R V$ morphological but without functional parameters. Not a single marker of fibrosis was independently associated with RV. MiR-30 was associated with RV impairment in the logistic regression model adjusted for age, sex, body mass index, and disease duration; however, lost its significance in the more comprehensive model.

Conclusions: Right ventricle structural and functional abnormalities are common in DCM. ECM fibrosis and serum markers are not associated with RV impairment. The prognostic value of studied microRNAs on RV is limited in DCM. (Cardiol J 2018; 25, 6: 722-731)

Key words: dilated cardiomyopathy, right ventricle, biopsy, extracellular matrix fibrosis, microRNA
\end{abstract}

Address for correspondence: Pawel Rubiś, MD, Department of Cardiac and Vascular Disease, John Paul II Hospital, ul. Prądnicka 80, 31-202 Kraków, Poland, tel: +48 126142 287, fax: +48 1242343 76, e-mail: pawelrub@poczta.onet.pl Received: 24.02 .2017 Accepted: 07.08.2017 


\section{Introduction}

Dilated cardiomyopathy (DCM) is primarily characterized by left ventricular (LV) wall thinning, chamber dilation and systolic dysfunction [1]. However, accompanying right ventricular (RV) involvement is frequently observed in DCM, and is related to poorer outcomes [2]. Precise measurement of LV morphology and function is relatively straightforward and based on solid geometric principles. On the other hand, due to its unique crescent shape, evaluation of RV size and function is far more demanding than its LV counterpart. Recent recommendations from the European Association of the Cardiovascular Imaging (EACVI) emphasize the importance of correct RV measurements [3].

Fibrosis of the extracellular matrix (ECM) is one of the hallmarks of DCM ultrastructural pathology. The gold standard is endomyocardial biopsy (EMB) and the detailed assessment of cardiac samples under a microscope. Taking another approach, an insight into the dynamics of ECM fibrosis can be provided with the assessment of blood levels of ECM metabolism markers, such as indices of collagen synthesis, fibrosis controlling factors, including osteopontin (OPN), transforming growth factor beta (TGF- $\beta$ ) or connective tissue growth factor (CTGF), and an antagonistic system of matrix metalloproteinases (MMPs) and their tissue inhibitors (TIMPs) [4].

Aside from these markers, there exist microRNAs: small (22 nucleotides) non-coding RNA sequences that bind to messenger RNA (mRNA) and inhibit protein translation or degrade mRNA. These are considered to be factors playing a vital role in the majority of cellular processes [5]. Among numerous candidates, up to the present, a set of five microRNAs: miR-21, miR-26, miR-29, miR-30, and miR-133a have been linked to cardiac fibrosis $[6,7]$. Additionally, we have recently compared expression of those circulating, fibrosis-linked microRNAs between DCM patients and healthy controls. Except for miR-133a, all of studied microRNAs were differently expressed in DCM compared to controls [7].

The left and right ventricles differ substantially in terms of morphology and function [8]. Similarly, molecular mechanisms leading to RV failure partly differ from those for LV pathology [9]. The relationship between $\mathrm{RV}$ abnormalities and ECM fibrosis, serum markers of fibrosis, and circulating fibrosis-linked microRNAs is largely unknown in DCM.
Therefore, the aim of the study was to test the hypothesis that the level of ECM fibrosis, serum levels of ECM metabolism markers and the profile of circulating microRNAs (miR-21, miR-26, miR-29, miR-30 and miR-133a) are dissimilar in DCM patients with and without RV abnormalities. Furthermore, we sought to uncover the associations between serum markers of ECM metabolism and circulating microRNAs with RV morphological and functional parameters.

\section{Methods}

\section{Study population}

From July 2014 to October 201570 consecutive DCM patients fulfilling pre-specified criteria and were willing to participate in the study were included. DCM was diagnosed in accordance with current recommendations having excluded significant coronary artery disease, primary heart valve disease, congenital heart disease, and arterial hypertension [1]. Based on detailed echocardiograms, all patients fulfilled strict morphological and functional criteria, e.g. all had dilated and significantly depressed systolic function (ejection fraction [EF] $<35 \%$ ) [10]. All patients had experienced stable heart failure (HF) symptoms, consistent with the New York Heart Association (NYHA) class I-III, for at least 2 weeks prior to the study. Disease duration was defined as the time elapsed from the first formal diagnosis of DCM to the point of recruitment in the present study. The study protocol was approved by the relevant institutional committees and the Ethical Committee. All patients gave written informed consent prior to inclusion in the study.

\section{Echocardiograpic assessment of RV morphology and function}

All measurements were performed according to the most recent recommendations of the EACVI [3]. Examinations were performed on commercially available equipment (Vivid 7 GE Medical System, Horten, Norway) with a phased-array of $1.5-4 \mathrm{MHz}$ transducer.

Based on recent EACVI guidelines, a conservative approach was followed and diagnosed RV dilatation (RVD) when both the basal RV dimension (RVd1) was above $41 \mathrm{~mm}$, and the mid-cavity RV dimension (RVd2) was $>35 \mathrm{~mm}$. In addition, $\mathrm{RV}$ systolic dysfunction (RVSD) was diagnosed if tricuspid annular plane systolic excursion (TAPSE) was below $17 \mathrm{~mm}$ and pulsed Doppler tissue imaging-derived tricuspid lateral annular systolic velocity $(\mathrm{RV}-\mathrm{S}$ ') $<9.5 \mathrm{~cm} / \mathrm{s}$ [3]. 


\section{Endomyocardial biopsy}

Endomyocardial biopsy procedures were performed by experienced operators via a femoral or jugular vein approach [11]. Long $(104 \mathrm{~cm})$, flexible, disposable biopsy forceps 7 French size with small jaws (Cordis ${ }^{\circledR}$, Johnson \& Johnson Co, Miami Lakes, FL, USA) were used for the procedure. Up to 5 myocardial samples were obtained from the RV septum, which were immediately stored in formalin for light microscopic examinations (2-3 samples) and snap-frozen in optimal cutting temperature-embedding medium and stored at $-80^{\circ} \mathrm{C}$ for further studies ( 2 samples). The presence of fibrosis was determined in 2-3 samples by an experienced pathologist blinded to the clinical data. Collagen volume fraction (CVF) was assessed by quantitative morphometry in biopsy sections stained with collagen specific picro-sirius red. CVF was defined as the percentage red-stained area per total myocardial tissue area [12].

\section{Serum markers of collagen metabolism}

Venous blood samples were drawn on the day of the study after a 30-min supine rest in a fasted state in the morning. After centrifuge, supernatant was stored at $-20^{\circ} \mathrm{C}$ until assay. The concentrations of collagen synthesis markers and markers of collagen degradation were determined in plasma using a commercially available ELISA tests as follows: collagen type 1, procollagen I N-terminal propeptide (PINP), procollagen III N-terminal propeptide (PIIINP), procollagen I C-terminal propeptide (PICP), procollagen III C-terminal propeptide (PIIICP), CTGF (all from Cloud Clone Corp. Houston, TX, USA); RayBio MMP2 ELISA, RayBio MMP9 ELISA, and RayBio TIMP-1 ELISA (all from RayBiotech, Norcross, GA, USA) and TGF- $\beta$ (Diaclone SAS, Besancon Cedex, France). All measurements were performed by technicians blinded to the sample status. Intra-assay and interassay coefficients of variation were $<7 \%$.

\section{Circulating microRNAs}

The quantitative polymerase chain reaction procedure used to measure microRNA levels and was performed as described previously [13]. RNA was extracted from $100 \mu \mathrm{L}$ of plasma using a MirVana kit (Life Technologies) following the manufacturer's protocol. $2 \mu \mathrm{L}$ of extracted RNA was used to perform reverse transcription with a TaqMan Advanced MicroRNA cDNA Synthesis Kit (Life Technologies). cDNA samples were diluted $10 \times$ before the qPCR reaction. qPCR was conducted on 384-well plates with TaqMan Advanced Mas-
terMix and TaqMan Advanced Assays targeting: hsa-miR-21-5p, hsa-mir-26a-5p, hsa-miR-29b-3p, hsa-miR-30c-5p, and hsa-miR-133a-3p. $15 \mu$ L reactions were prepared with pipetting station Bravo (Agilent Technologies) and a real-time reaction was run and read on a CFX384 Real Time PCR Detection System (Bio-Rad). Mean Cq values were normalized to the geometric mean of hsa-miR-15b-5p and hsa-miR-16-5p, which were selected as relatively stable controls in pilot experiments. Normalized data was expressed for each sample as $\left[2^{-\Delta \mathrm{C} q}\right]$ where $\Delta \mathrm{Cq}$ is the difference between the $\mathrm{Cq}$ value between the microRNA of interest and the geometric mean of miR-15b and miR-16 for a particular sample.

\section{Statistical analysis}

The distribution of variables was assessed with the Shapiro-Wilk test. Comparisons of clinical parameters within groups were conducted with the t-Student test or with Mann-Whitney tests if any lack of normality was found therein. Univariate relationships between RV parameters and serum markers of fibrosis and microRNAs were determined by Pearson correlation analysis. Associations between serum markers of fibrosis and circulating microRNAs with RV morphological and functional parameters were analyzed with logistic regression methods. Three logistic regression models were analyzed: an unadjusted model ( $1^{\text {st }}$ model), a model with adjustments for age, sex, body mass index (BMI) and disease duration ( $2^{\text {nd }}$ model), and the most comprehensive model, with adjustments for age, sex, BMI, disease duration, EF, right atrium (RA) area, ECM fibrosis, pulmonary hypertension, and $\mathrm{N}$-terminal $\mathrm{B}$-type natriuretic peptide (NT-proBNP) ( ${ }^{\text {rd }}$ model). All results were considered statistically significant when $\mathrm{p}$ was $<0.05$. The entire analysis was performed using the SPSS package, version 14.0 (SPSS Inc., Chicago, Illinois) and R 3.1.2 (R Foundation for Statistical Computing, Vienna, Austria).

\section{Results}

\section{Baseline characteristics}

Based on RV measurements, patients were divided into two groups: those with normal RV dimensions and preserved systolic function (group $1, \mathrm{n}=15,21.4 \%$ ), and those with RVD and/or RVSD (group 2, $\mathrm{n}=55,78.6 \%$ ). Table 1 shows the baseline characteristics of these two groups. Patients with normal RV were found to have a significantly shorter duration of disease compared to 
Table 1. Baseline characteristics of the study population, divided into those with normal right ventricle dimensions and systolic function (group 1), and those with right ventricle dilatation and/or systolic dysfunction (group 2).

\begin{tabular}{|c|c|c|c|}
\hline Parameter & $\begin{array}{c}\text { Group } 1 \\
\text { (n }=15,21.4 \%)\end{array}$ & $\begin{array}{c}\text { Group } 2 \\
(\mathrm{n}=55,78.6 \%)\end{array}$ & $\mathbf{P}$ \\
\hline Age [years] & $45.6 \pm 12.1$ & $48.7 \pm 12.1$ & 0.38 \\
\hline Sex [male/female] & $12(80 \%) / 3(20 \%)$ & $51(92.7 \%) / 4(7.3 \%)$ & 0.14 \\
\hline BMI $\left[\mathrm{kg} / \mathrm{m}^{2}\right]$ & $27.9 \pm 6.5$ & $27 \pm 5$ & 0.54 \\
\hline NYHA class & $2.47 \pm 0.83$ & $2.6 \pm 0.71$ & 0.54 \\
\hline Duration of disease [months] & $4.1 \pm 5.7$ & $29.8 \pm 38.3$ & $<0.001$ \\
\hline QRS [ms] & $102.7 \pm 36.3$ & $112.1 \pm 32.6$ & 0.34 \\
\hline LVESd/BSA $\left[\mathrm{mm} / \mathrm{m}^{2}\right]$ & $26.6 \pm 5.1$ & $31 \pm 7.3$ & 0.03 \\
\hline LVEDd/BSA [mm/m²] & $32.7 \pm 3.7$ & $36.4 \pm 7.5$ & 0.01 \\
\hline LVESvol/BSA [mL/m²] & $79.6 \pm 39.1$ & $100.1 \pm 50.6$ & 0.18 \\
\hline LVEDvol/BSA [mL/m²] & $111.8 \pm 47.9$ & $130.4 \pm 62.1$ & 0.32 \\
\hline Ejection fraction [\%] & $26.8 \pm 6.6$ & $23.7 \pm 7.5$ & 0.15 \\
\hline$E / E^{\prime}$ (average sep+lat) & $15.6 \pm 5.9$ & $21.5 \pm 12.2$ & 0.02 \\
\hline RVOT prox [mm] & $28.7 \pm 6.7$ & $32.9 \pm 7.3$ & 0.06 \\
\hline $\mathrm{RVd} 1[\mathrm{~mm}]$ & $30.2 \pm 4.9$ & $37.3 \pm 8.2$ & 0.007 \\
\hline $\mathrm{RVd} 2[\mathrm{~mm}]$ & $24.9 \pm 6.9$ & $33.7 \pm 7.9$ & 0.001 \\
\hline $\mathrm{RVd} 3[\mathrm{~mm}]$ & $76.2 \pm 7.2$ & $83.8 \pm 15.8$ & 0.1 \\
\hline RV thickness [mm] & $3.7 \pm 1.1$ & $3.9 \pm 1.1$ & 0.67 \\
\hline TAPSE [mm] & $23.6 \pm 6.1$ & $15.3 \pm 3.9$ & 0.008 \\
\hline RV-S' $[\mathrm{cm} / \mathrm{s}]$ & $11.8 \pm 3.2$ & $7.6 \pm 2.8$ & $<0.001$ \\
\hline LA area $\left[\mathrm{cm}^{2}\right]$ & $26.7 \pm 9.3$ & $31.7 \pm 8.2$ & 0.05 \\
\hline $\mathrm{RA}$ area $\left[\mathrm{cm}^{2}\right]$ & $19.9 \pm 5.4$ & $25.2 \pm 8$ & 0.05 \\
\hline PASP [mmHg] & $33.4 \pm 7.7$ & $41.5 \pm 16.7$ & 0.01 \\
\hline ECM fibrosis & $4(26.7 \%)$ & $20(36.4 \%)$ & 0.48 \\
\hline CVF [\%] & $5.3 \pm 3.4$ & $6 \pm 6.5$ & 0.83 \\
\hline $\mathrm{Cl}\left[\mathrm{mL} / \mathrm{min} / \mathrm{m}^{2}\right]$ & $2.3 \pm 0.5$ & $1.9 \pm 0.46$ & 0.002 \\
\hline PA mean $[\mathrm{mmHg}]$ & $17.4 \pm 5.4$ & $24.4 \pm 11.4$ & 0.002 \\
\hline PA saturation [\%] & $68.4 \pm 4.3$ & $61.7 \pm 8.7$ & $<0.001$ \\
\hline PCWP mean [mmHg] & $10.5 \pm 6.3$ & $16.6 \pm 8.3$ & 0.02 \\
\hline Ao mean [mmHg] & $91.3 \pm 10.8$ & $89.7 \pm 14.5$ & 0.71 \\
\hline Pulmonary hypertension & $1(7.1 \%)$ & $26(47.3 \%)$ & 0.006 \\
\hline $\mathrm{VO}_{2}$ peak $[\mathrm{mL} / \mathrm{kg} / \mathrm{min}]$ & $19 \pm 5.5$ & $16.2 \pm 6.1$ & 0.33 \\
\hline Hemoglobin [g/dL] & $14.5 \pm 1.6$ & $14.4 \pm 1.6$ & 0.96 \\
\hline hs-troponin T [ng/mL] & $0.0145 \pm 0.0096$ & $0.0243 \pm 0.0194$ & 0.09 \\
\hline NT-proBNP [pg/mL] & $2881.3 \pm 7006$ & $3503.5 \pm 5005$ & 0.71 \\
\hline Beta-blocker & $15(100 \%)$ & $54(98.2 \%)$ & 0.6 \\
\hline ACE-I or ARB & $15(100 \%)$ & $54(98.2 \%)$ & 0.6 \\
\hline MRA & $15(100 \%)$ & $51(92.7 \%)$ & 0.28 \\
\hline CRT-D & $2(13.3 \%)$ & $25(45.5 \%)$ & 0.02 \\
\hline
\end{tabular}

BMI - body mass index; NYHA - New York Heart Association class; LVESd/BSA — left ventricular end-systolic diameter indexed to body surface area; LVEDd/BSA — left ventricular end-diastolic diameter indexed to body surface area; LVESvol/BSA — left ventricular end-systolic volume indexed to body surface area; LVEDvol/BSA - left ventricular end-diastolic volume indexed to body surface area; E/E' (average sep+lat) - ratio of early mitral inflow $E$-wave and early myocardial $E^{\prime}$ velocity ( $E^{\prime}$ - is an average of septal and lateral myocardial velocity); RVOT - right ventricular outflow tract; RVd1;2;3 - right ventricular measurements from the apical four-chamber view; TAPSE - tricuspid annular plane systolic excursion; RV-S' - pulsed DTI-derived tricuspid lateral annular systolic velocity; LA area/RA area - left and right atrial area; PASP — pulmonary artery systolic pressure; ECM fibrosis — extracellular matrix fibrosis; CVF — collagen volume fraction; Cl - cardiac index; PA mean - mean pulmonary artery pressure; PCWP - pulmonary artery wedge pressure; Ao mean - mean aortic pressure; $\mathrm{O}_{2}$ peak - peak oxygen uptake; hs-troponin T — high sensitivity troponin T; NT-proBNP — N-terminal pro B-type natriuretic peptide; ACE-I - angiotensin converting enzyme inhibitor; ARB — angiotensin receptor type 1 blocker; MRA — mineralocorticoid receptor antagonist; CRT-D cardiac resynchronization therapy with cardioverter-defibrillator 
Table 2. Comparison of serum markers of fibrosis between dilated cardiomyopathy patients with normal and abnormal right ventricle.

\begin{tabular}{lccc}
\hline Parameter & Group $1(\mathbf{n}=15,21.4 \%)$ & Group 2 $(\mathbf{n}=55,78.6 \%)$ & $P$ \\
\hline PICP $[\mathrm{ng} / \mathrm{mL}]$ & $0.15(0.08-0.28)$ & $0.18(0.06-0.46)$ & 0.67 \\
PINP $[\mathrm{pg} / \mathrm{mL}]$ & $125.4(78.4-380.1)$ & $131(25.2-1050)$ & 0.69 \\
PIIICP $[\mathrm{pg} / \mathrm{mL}]$ & $261.1(87.2-1165)$ & $195.7(70.3-937.2)$ & 0.2 \\
PIIINP $[\mathrm{ng} / \mathrm{mL}]$ & $4.4(2.4-5.1)$ & $4.4(2.2-6.1)$ & 0.92 \\
Col-1 $[\mathrm{pg} / \mathrm{mL}]$ & $83.4(35.3-206.5)$ & $56.4(23.1-233.4)$ & 0.13 \\
OPN $[\mathrm{ng} / \mathrm{mL}]$ & $2.9(1.87-7.91$ & $3.18(0.74-19.4)$ & 0.5 \\
TGF- $\beta 1[\mathrm{ng} / \mathrm{mL}]$ & $2.71(1.36-5.73)$ & $2.2(0.65-6.9)$ & 0.3 \\
CTGF $[\mathrm{ng} / \mathrm{mL}]$ & $3.56(1.21-12.4)$ & $3.8(0.5-23.9)$ & 0.61 \\
MMP-2 $[\mathrm{ng} / \mathrm{mL}]$ & $5.9(2.9-7.4)$ & $6.1(2.7-14.8)$ & 0.23 \\
MMP-9 $[\mathrm{ng} / \mathrm{mL}]$ & $2.2(0.28-5.2)$ & $1.9(0.3-9.2)$ & 0.81 \\
TIMP-1 $[\mathrm{ng} / \mathrm{mL}]$ & $15.3(1.9-32.5)$ & $15.1(1.8-36.2)$ & 0.56 \\
\hline
\end{tabular}

Data are presented as median (range); PICP — procollagen I C-terminal propeptide; PINP — procollagen I N-terminal propeptide; PIIICP — procollagen III C-terminal propeptide; PIIINP — procollagen III N-terminal propeptide; Col-1 — collagen type 1; OPN — osteopontin; $\mathrm{TGF} \beta$ - transforming growth factor beta; CTGF — connective tissue growth factor; MMP — matrix metalloproteinase; TIMP - tissue inhibitor of matrix metalloproteinase

Table 3. Comparison of circulating microRNAs between dilated cardiomyopathy patients with normal and abnormal right ventricle.

\begin{tabular}{lccc}
\hline Parameter & Group $\mathbf{1}(\mathbf{n}=\mathbf{1 5})$ & Group 2 ( $=55)$ & P \\
\hline mir-21 $[\Delta \mathrm{Cq}]$ & $0.19 \pm 0.48$ & $0.12 \pm 0.64$ & 0.69 \\
mir-21 $\left[2^{-\Delta C q}\right]$ & $0.92 \pm 0.27$ & $1.01 \pm 0.49$ & 0.48 \\
mir-26 $[\Delta C q]$ & $-0.102 \pm 0.87$ & $-0.032 \pm 0.78$ & 0.77 \\
mir-26 $\left[2^{-\Delta C q}\right]$ & $1.25 \pm 0.69$ & $1.18 \pm 0.67$ & 0.7 \\
mir-29 $[\Delta C q]$ & $3.03 \pm 0.61$ & $2.72 \pm 0.8$ & 0.17 \\
mir-29 $\left[2^{-\Delta C q}\right]$ & $0.13 \pm 0.05$ & $0.17 \pm 0.088$ & 0.1 \\
mir-30a $[\Delta C q]$ & $8.43 \pm 1.95$ & $7.64 \pm 1.57$ & 0.12 \\
mir-30a $\left[2^{-\Delta C}\right]$ & $0.82 \pm 0.018$ & $0.9 \pm 0.011$ & 0.84 \\
mir-133a $[\Delta C q]$ & $8.05 \pm 1.48$ & $7.38 \pm 2.47$ & 0.32 \\
mir-133a $\left[2^{-\Delta C q}\right]$ & $\mathbf{0 . 6} \pm \mathbf{0 . 0 7}$ & $\mathbf{1 . 4 1} \pm \mathbf{0 . 2}$ & $\mathbf{0 . 0 0 6}$ \\
\hline
\end{tabular}

Data are presented as mean \pm standard deviation

those with RVD and/or RVSD (4.1 \pm 5.7 vs. $29.8 \pm$ \pm 38.3 months, $\mathrm{p}<0.001$, respectively). Those patients with impaired RV had significantly worse LV diastolic function. Not surprisingly, RV basal and mid-cavity diameters (RVd1 and RVd2) were significantly enlarged in group 2 patients as were indices of RV systolic function - TAPSE and RVS'; nonetheless, RV outflow tract (RVOT) proximal diameter, RV longitudinal diameter (RVd3), and RV free wall thickness were found to be similar in both groups. Patients from group 2 were more often diagnosed with secondary pulmonary hypertension. ECM fibrosis as well as the magnitude of fibrosis, as quantified with CVF, were evenly distributed between the two groups.
Comparison of markers of fibrosis and microRNAs between patients with normal and impaired RV

There were no differences in all studied serum markers of fibrosis between the two groups (Table 2), whereas out of the five microRNAs under study, only miR-133a in $\left[2^{-\mathrm{DCq}}\right]$ units were differently expressed in patients with normal and abnormal RV (Table 3).

\section{Correlations between markers} of ECM fibrosis and RV parameters

$\mathrm{RVd} 1$ and RVd2, correlated with OPN and MMP-2 or MMP-2 and TIMP-1, respectively (Table 4). Interestingly, the remaining RV ana- 


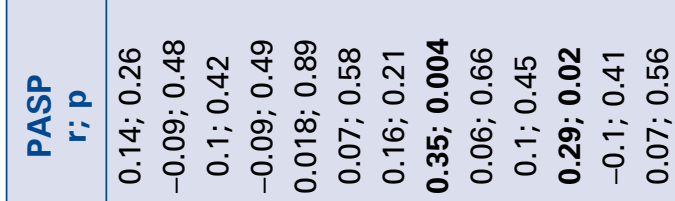

ป⿻一𠃋十 \に

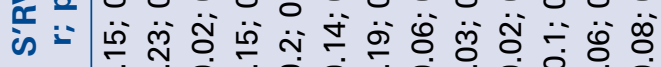

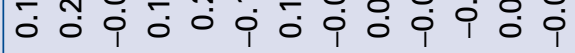

U

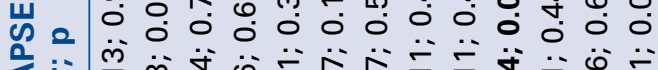

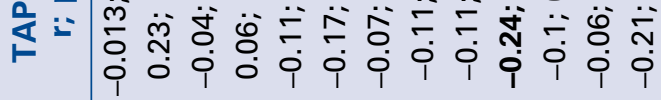
ॠ \%

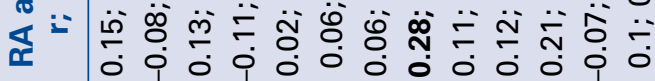
\&

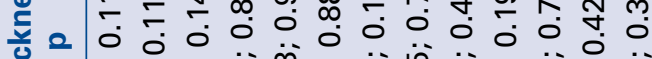

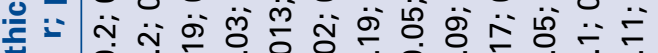
$\geqslant$ †

m 응 \= i $\begin{aligned} & 0 \\ & 0\end{aligned}$ i

ำ 누

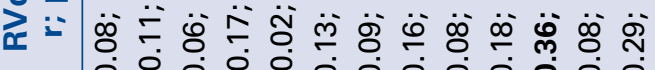

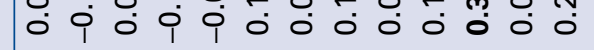

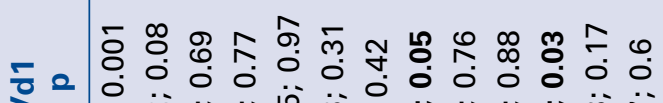
I

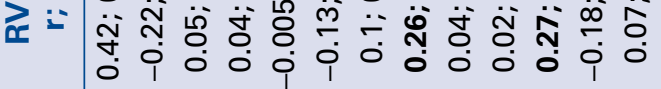
ᄂ = 任

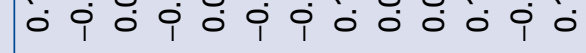

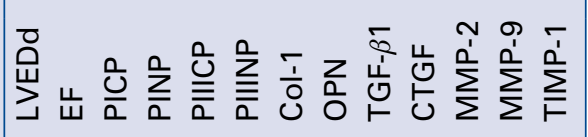

\begin{tabular}{|c|c|}
\hline $\begin{array}{l}0 \\
\bar{c} \\
\vdots\end{array}$ & 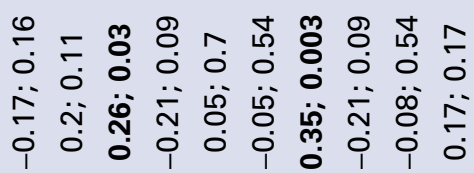 \\
\hline 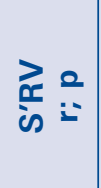 & 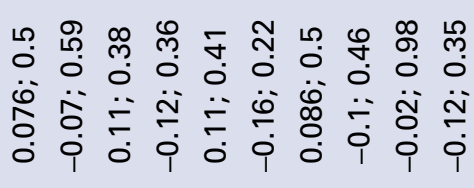 \\
\hline 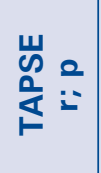 & 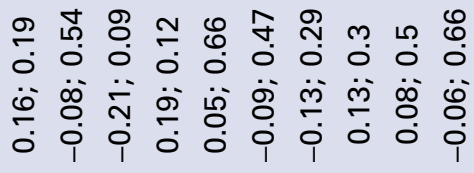 \\
\hline 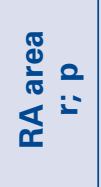 & 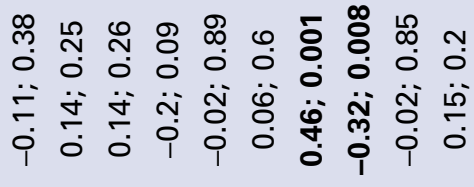 \\
\hline 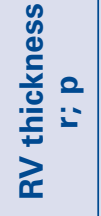 & 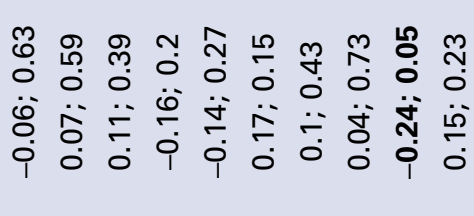 \\
\hline $\begin{array}{l}2 \\
\therefore\end{array}$ & 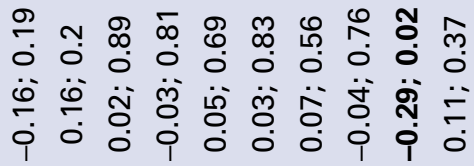 \\
\hline$\sum_{x}^{x}:$ & 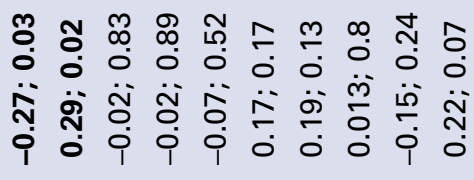 \\
\hline & 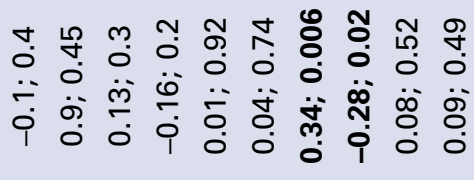 \\
\hline $8 \div$ & 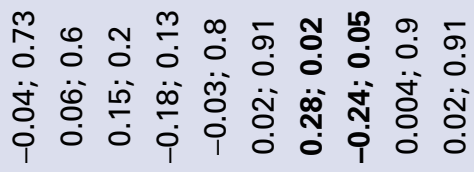 \\
\hline & 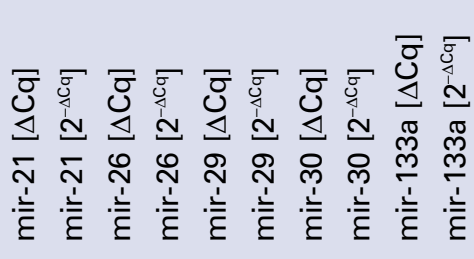 \\
\hline
\end{tabular}


tomical parameters, including RVOT proximal diameter, RVd3, and RV wall thickness, were not seen to correlate with any marker of fibrosis. As for RV systolic function parameters, only TAPSE correlated with CTGF; in contrast to this, RV-S' showed no correlation with any marker of fibrosis. Finally, pulmonary artery systolic pressure (PASP) correlated with OPN and MMP-2. It should be noted that not a single marker of collagen synthesis (PICP, PINP, PIIICP, PIIINP, and col-1) correlated with any RV parameter.

\section{Correlations between circulating microRNAs and RV parameters}

In terms of RV anatomical parameters, miR-30 correlated with RVOT proximal diameter, RVd1 and RA area (Table 5). Additionally, miR-21 correlated with $\mathrm{RVd} 2$, and miR-133a correlated with $\mathrm{RVd} 3$ and RV wall thickness. Importantly, no microRNA correlated with RV systolic function parameters, including TAPSE and RV-S'.

\section{Associations between markers of ECM}

fibrosis and microRNAs with RV parameters

It was verified as to whether there were any independent associations between serum markers of fibrosis and circulating microRNAs with RV parameters. There were no markers of fibrosis independently associated with RV morphological and functional parameters (Table 6). Out of the five microRNAs studied, only miR-30 was associated with RV impairment (OR 0.41; p < 0.02) in the $2^{\text {nd }}$ model (Table 7). However, this link was no longer observed in the $3^{\text {rd }}$ model, which had been adjusted for more powerful RV-associated variables.

\section{Discussion}

The distribution of ECM fibrosis as well as the magnitude of fibrosis was similar in patients with and without RV abnormalities. Second, no differences in serum levels of ECM metabolism markers were observed between DCM patients with and without RVD and/or RVSD. Thirdly, expression levels of only one out of five fibrosis-linked circulating microRNAs - namely miR-133a - was different when comparing patients with and without RV abnormalities. Fourthly, RV morphological and functional parameters correlated with only a few markers of ECM fibrosis, including OPN and CTGF and MMPs/TIMPs system. Remarkably, none of the $\mathrm{RV}$ parameters correlated with markers of collagen synthesis. In addition, it was found that circulating microRNAs correlated with RV morphological indices; however, no correlations were observed between microRNAs and RV systolic function parameters, including TAPSE and RV-S'. Not a single marker of ECM fibrosis was independently associated with RV abnormalities. Finally, only mir-30 was associated with RV impairment in the logistic regression model adjusted for age, sex, BMI, and disease duration; however, this association was no longer observed in the $3^{\text {rd }}$ model which was additionally adjusted for EF, RA area, ECM fibrosis, pulmonary hypertension, and NT-proBNP.

Associations between ECM fibrosis, markers of ECM metabolism and RV parameters

Before more detailed results can be interpreted, it is important to know the relations of serum markers of ECM metabolism between DCM and controls. It was previously presented that among markers of collagen synthesis, only PIIINP was significantly higher in DCM, whereas PICP, PINP, and PIIICP were similar in DCM and controls. OPN and CTGF levels were higher in DCM but levels of TGF- $\beta 1$ did not differ between DCM and controls. MMP-2, MMP-9 and TIMP-1 were significantly higher in DCM [14]. There are no studies directly addressing the relationship between ECM fibrosis and serum markers of ECM metabolism with RV morphology and function in DCM. Past studies have shown that the percentage of myocardial collagen type I, III and IV significantly differs between LV and RV in DCM, and the same has been discovered for expressions of MMP-1, MMP-2, and MMP-9 [15]. These established findings support the hypothesis that distinct patterns of ECM structure, and particular turnover pathways, exist in the RV and LV [16]. Further empirical evidence for this was provided by Tyagi et al. [17], who observed differences in collagenolytic MMPs activity between $\mathrm{LV}$ and $\mathrm{RV}$, both after myocardial infarction and in DCM. Another result found that LV unloading, with LV assist devices, decreased $\mathrm{RV}$ total collagen and myocardial TNF- $\alpha$ content. Thus, a decrease in ECM fibrosis and normalization of cytokine up-regulation is an important effect of mechanical unloading associated with both LV and $\mathrm{RV}$ recovery [18]. Present observations report a lack of associations between ECM fibrosis, serum markers of fibrosis and RV morphology and function. In endeavoring to explain these findings, the following was postulated. On the one hand, it may be that the serum markers of ECM fibrosis under study are imperfect tools for a reliable assessment of dynamics of ECM fibrosis; on the other hand, substantial differences were observed in those 
Table 6. Value of serum markers of fibrosis in predicting right ventricle dilatation and/or systolic dysfunction in an unadjusted ( $1^{\text {st }}$ model) and adjusted models: ( $2^{\text {nd }}$ model) adjusted for age, sex, body mass index and disease duration, and (the $3^{\text {rd }}$ model) additionally adjusted for ejection fraction, right atrial area, extracellular matrix fibrosis, pulmonary hypertension, and NT-proBNP.

\begin{tabular}{lccc}
\hline Parameter & Model 1 & Model 2 & Model 3 \\
\hline PICP $[\mathrm{ng} / \mathrm{mL}]$ & OR $=0.834 ; p=0.514$ & OR $=0.935 ; p=0.852$ & OR $=0.062 ; p=0.629$ \\
PINP $[\mathrm{pg} / \mathrm{mL}]$ & OR $=0.99993 ; p=0.971$ & OR $=0.998 ; p=0.554$ & OR $=0.487 ; p=0.996$ \\
PIIICP $[\mathrm{ng} / \mathrm{mL}]$ & OR $=0.29 ; p=0.328$ & OR $=0.499 ; p=0.68$ & OR $=0.001 ; p=0.554$ \\
PIIINP $[\mathrm{pg} / \mathrm{mL}]$ & OR $=1.00007 ; p=0.837$ & OR $=1.00006 ; p=0.907$ & OR $\geq 100 ; p=0.999$ \\
Col-1 $[\mathrm{pg} / \mathrm{mL}]$ & OR $=0.99735 ; p=0.075$ & OR $=0.998 ; p=0.368$ & OR $\leq 0.00001 ; p=0.999$ \\
OPN $[\mathrm{ng} / \mathrm{mL}]$ & OR $=0.994 ; p=0.953$ & OR $=0.908 ; p=0.737$ & OR $\leq 0.00001 ; p=1$ \\
TGF- $\beta 1[\mathrm{pg} / \mathrm{mL}]$ & OR $=0.99975 ; p=0.282$ & OR $=1.00002 ; p=0.947$ & OR $=1.502 ; p=0.99$ \\
CTGF $[\mathrm{ng} / \mathrm{mL}]$ & OR $=1.025 ; p=0.784$ & OR $=1.015 ; p=0.916$ & OR $\geq 100 ; p=0.91$ \\
MMP-2 $[\mathrm{ng} / \mathrm{mL}]$ & OR $=1.249 ; p=0.203$ & OR $=1.246 ; p=0.346$ & OR $\geq 100 ; p=0.998$ \\
MMP-9 $[\mathrm{pg} / \mathrm{ml}]$ & OR $=1 ; p=0.98$ & OR $=1.00007 ; p=0.75$ & OR $=1.137 ; p=0.993$ \\
TIMP-1 $[\mathrm{pg} / \mathrm{mL}]$ & OR $=1.00002 ; p=0.488$ & OR $=1.00002 ; p=0.597$ & OR $=1.033 ; p=0.991$ \\
\hline
\end{tabular}

NT-proBNP - N-terminal pro B-type natriuretic peptide; OR — odds ratio; other abbreviations - see Table 2

Table 7. Value of serum markers of fibrosis in predicting right ventricle dilatation and/or systolic dysfunction in an unadjusted ( $1^{\text {st }}$ model $)$ and adjusted models: $\left(2^{\text {nd }}\right.$ model $)$ adjusted for age, sex, body mass index and disease duration, and (the $3^{\text {rd }}$ model) additionally adjusted for ejection fraction, right atrial area, extracellular matrix fibrosis, pulmonary hypertension, and NT-proBNP.

\begin{tabular}{|c|c|c|c|}
\hline Parameter & Model 1 & Model 2 & Model 3 \\
\hline mir-21 $[\Delta \mathrm{Cq}]$ & $\mathrm{OR}=0.825 ; p=0.694$ & $\mathrm{OR}=0.443 ; p=0.211$ & $\mathrm{OR}=0.005 ; p=0.628$ \\
\hline $\operatorname{mir}-21\left[2^{-\Delta C q}\right]$ & $O R=1.708 ; p=0.471$ & $O R=5.011 ; p=0.156$ & $O R \geq 100 ; p=0.494$ \\
\hline $\operatorname{mir}-26[\Delta \mathrm{Cq}]$ & $\mathrm{OR}=1.118 ; p=0.763$ & $O R=0.905 ; p=0.87$ & $O R \geq 100 ; p=0.995$ \\
\hline $\operatorname{mir}-26\left[2^{-\Delta C q}\right]$ & $\mathrm{OR}=0.848 ; \mathrm{p}=0.698$ & $O R=1.157 ; p=0.842$ & $\mathrm{OR} \leq 0.00001 ; p=0.994$ \\
\hline $\operatorname{mir}-29[\Delta \mathrm{Cq}]$ & $\mathrm{OR}=0.612 ; \mathrm{p}=0.19$ & $\mathrm{OR}=0.672 ; \mathrm{p}=0.523$ & $\mathrm{OR} \leq 0.00001 ; p=0.997$ \\
\hline $\operatorname{mir}-29\left[2^{-\Delta C q}\right]$ & $O R \geq 100 ; p=0.11$ & $\mathrm{OR}=36.439 ; p=0.549$ & $O R \geq 100 ; p=0.997$ \\
\hline $\operatorname{mir}-30[\Delta \mathrm{Cq}]$ & $O R=0.738 ; p=0.125$ & $\mathrm{OR}=0.41 ; p=0.023^{*}$ & $\mathrm{OR} \leq 0.00001 ; \mathrm{p}=0.999$ \\
\hline $\operatorname{mir}-30\left[2^{-\Delta C q}\right]$ & $O R \geq 100 ; p=0.835$ & $O R \geq 100 ; p=0.067$ & $O R \geq 100 ; p=0.999$ \\
\hline $\operatorname{mir}-133 a[\Delta \mathrm{Cq}]$ & $\mathrm{OR}=0.883 ; p=0.319$ & $\mathrm{OR}=0.83 ; p=0.336$ & $O R \geq 100 ; p=0.974$ \\
\hline $\operatorname{mir}-133 a\left[2^{-\Delta C q}\right]$ & $O R \geq 100 ; p=0.12$ & $O R \geq 100 ; p=0.29$ & $O R \geq 100 ; p=0.999$ \\
\hline
\end{tabular}

$*<0.05$

markers between DCM and controls. Although ECM turnover is clearly increased in DCM, no direct links between ECM fibrosis and RV impairment were observed. This observation is important as it helps to understand why specific anti-fibrotic therapies, tested in HF and DCM, did not translate into substantial improvements in RV morphology and function. Furthermore, the fact that the percentage of patients with fibrosis is similar whether with and without RV abnormalities which may suggest that once ECM fibrosis starts (probably very early on in the course of the disease) it is homogenously increased in all DCM patients.

Associations between circulating microRNAs and RV morphology and function

As the process of $\mathrm{LV}$ and RV adaptation to stress and loading conditions differ substantially, chamber-specific microRNAs patterns are to be expected. In a recent study, Kakimoto et al. [19] showed prominent micro-RNA chamber-specificity, and found that, out of 438 microRNAs studied, 
25 were differentially expressed when comparing the LA and LV. In line with this, Zhang et al. [20] reported that 169 microRNAs were expressed at different levels in the human RA and RV myocardium. Unfortunately, there are no studies directly comparing the microRNA profile between LV and RV. Still, recent studies have shown that circulating microRNAs reflect tissue microRNAs. De Rosa et al. [21] and Melman et al. [22], who studied transcoronary gradients of microRNAs levels, provided evidence that miR-133 and miR-30 are directly released from the heart. Li et al. [23] showed that 36 microRNAs were expressed differently in patients with a ventricular septal defect compared to controls, and the majority of those microRNAs targeted genes related to RV morphogenesis. Also, Sucharow et al. [24] observed different microRNA profiles in pediatric patients with hypoplastic left heart syndrome in comparison to pediatric and adult patients with DCM. Similarly, Lai et al. [25] found 11 microRNAs that were significantly increased in patients after an atrial switch operation for complete transposition of the great arteries (TGA) compared to healthy controls. In contrast to these findings, Tutarel et al. [26] did not observe any differences in levels of circulating miR-423-5p between patients with a systemic RV and reduced EF after atrial repair for TGA and controls. In another study, Satoh et al. [27] reported an association between miR-208, cardiac fibrosis and outcomes in DCM, but no associations between microRNAs and ECM fibrosis.

Surprisingly, a comparison of the expression levels of fibrosis-linked microRNAs in DCM patients with and without RV morphological and/ /or functional abnormalities has not yet been performed. Thus, reported herein for the first time, that miR-133a was the sole microRNA expressed differently in patients with preserved and abnormal RV. All microRNAs, except for miR-29, correlated with various RV diameters, RA area, and PASP. Importantly, none of the microRNAs correlated with RV systolic function parameters. Current research indicates that microRNAs may also serve as predictive parameters, as confirmed by Devaux et al. [28] who reported that a panel of four microRNAs, including miR-16, miR-27a, miR-101 and miR-150 improved the prediction of LV contractility in patients after acute myocardial infarction. In addition, it was recently observed that following circulating microRNAs: miR-3135b, mir-3908 and miR-5571-5p may also serve as potential as diagnostic markers in DCM [29]. Therefore, in order to clarify the as-yet-ambigu- ous issue of the relationship between the various microRNAs, we researched RV morphological and functional parameters, the logistic regression analyses assessed the added predictive value of these microRNAs against multi-parameter clinical models, including established predictors of RV morphology and function. The results obtained showed that miR-30 alone was associated with $\mathrm{RV}$ impairment in unadjusted model but lost its significance in the more comprehensive model.

\section{Limitations of the study}

Although we sampled up to five biopsies along the RV interventricular septum, the presence of fibrosis was assessed in 2-3 samples, thus, the possibility cannot excluded that, due to the patchy distribution of fibrosis, this assessment may not be completely accurate. On the other hand, ECM fibrosis was confirmed in $24(34.3 \%)$ patients and CVF was $5.88 \pm 6.06 \%$ - findings that are consistent with other studies. The distribution of patients with normal and abnormal RV was uneven ( 15 vs. 55 patients), which may influence the robustness of statistical analyses. Patients who developed abnormal RV suffered from DCM for a significantly longer period than those with normal RV (29.8 \pm \pm 38.3 vs. $4.1 \pm 5.7$ months, $\mathrm{p}<0.001$, respectively). Consequently, those patients had significantly increased incidence of CRT-D implantation (25 [45.5\%] with RVD and/or RVSD vs. 2 [13.3\%] without RVD and/or RVSD, $p<0.02$, respectively), which is a proven intervention that reverses left and right ventricular remodeling.

\section{Conclusions}

Right ventricle dilation and/or systolic dysfunction were common and were observed in more than three-quarters of the DCM cohort. Invasively-determined ECM fibrosis as well as serum markers of fibrosis are not useful in predicting RV abnormalities. The expression of circulating microRNAs, with the exception of miR-133a, was similar in DCM patients regardless of RV dilation and/or systolic impairment. The microRNAs miR-21, miR-26, miR-30a, and miR-133a correlated with various $\mathrm{RV}$ morphological parameters; however, none of the microRNAs correlated with RV systolic function indices. Mir-30 was associated with RV impairment in the logistic regression model adjusted for age, sex, BMI, and disease duration; however, this association was no longer observed in the most comprehensive model that was adjusted for more powerful RV-associated 
variables. The prognostic value of the microRNAs researched in this paper on RV morphology and function is limited in DCM.

\section{Acknowledgements}

This work was founded through the $\mathrm{Na}$ tional Science Centre, Poland (grant 2013/09/D/ NZ5/00252) and the Department of Scientific Research and Structural Funds of Medical College, Jagiellonian University (grant K/ZDS/004596).

\section{Conflict of interest: None declared}

\section{References}

1. Elliott P, Andersson B, Arbustini E, et al. Classification of the cardiomyopathies: a position statement from the european society of cardiology working group on myocardial and pericardial diseases. Eur Heart J. 2007; 29(2): 270-276, doi: 10.1093/eurheartj/ehm342.

2. Mahmud M, Champion HC. Right ventricular failure complicating heart failure: pathophysiology, significance, and management strategies. Curr Cardiol Rep. 2007; 9(3): 200-208, indexed in Pubmed: 17470333.

3. Lang RM, Badano LP, Mor-Avi V, et al. Recommendations for cardiac chamber quantification by echocardiography in adults: an update from the American Society of Echocardiography and the European Association of Cardiovascular Imaging. J Am Soc Echocardiogr. 2015; 28(1): 1-39.e14, doi:10.1016/j.echo.2014.10.003, indexed in Pubmed: 25559473.

4. Sackner-Bernstein JD, Sackner-Bernstein JD. The myocardial matrix and the development and progression of ventricular remodeling. Curr Cardiol Rep. 2000; 2(2): 112-119, indexed in Pubmed: 10980881.

5. Zhang Y, Tang W, Peng L, et al. Identification and validation of microRNAs as endogenous controls for quantitative polymerase chain reaction in plasma for stable coronary artery disease. Cardiol J. 2016; 23(6): 694-703, doi: 10.5603/CJ.2016.0109, indexed in Pubmed: 27976798.

6. Vegter EL, van der Meer P, de Windt LJ, et al. MicroRNAs in heart failure: from biomarker to target for therapy. Eur J Heart Fail. 2016; 18(5): 457-468, doi: 10.1002/ejhf.495, indexed in Pubmed: 26869172.

7. Rubiś P, Totoń-Żurańska J, Wiśniowska-Śmiałek S, et al. Relations between circulating microRNAs (miR-21, miR-26, miR-29, miR-30 and miR-133a), extracellular matrix fibrosis and serum markers of fibrosis in dilated cardiomyopathy. Int J Cardiol. 2017; 231: 201-206, doi:10.1016/j.ijcard.2016.11.279, indexed in Pubmed: 27889210.

8. Majos E, Dąbrowski R, Szwed H. The right ventricle in patients with chronic heart failure and atrial fibrillation. Cardiol J. 2013; 20(3): 220-226, doi:10.5603/CJ.2013.0065, indexed in Pubmed: 23788294.

9. Reddy S, Bernstein D. Molecular Mechanisms of Right Ventricular Failure. Circulation. 2015; 132(18): 1734-1742, doi:10.1161/CIRCULATIONAHA.114.012975, indexed in Pubmed: 26527692.

10. Henry WL, Gardin JM, Ware JH. Echocardiographic measurements in normal subjects from infancy to old age. Circulation. 1980; 62(5): 1054-1061, indexed in Pubmed: 7418156.

11. Cooper LT, Baughman KL, Feldman AM, et al. The role of endomyocardial biopsy in the management of cardiovascular disease: a scientific statement from the American Heart Association, the American College of Cardiology, and the European Society of Cardiology. Circulation. 2007; 116(19): 2216-2233, doi: 10.1161/CIRCULATIONAHA.107.186093, indexed in Pubmed: 17959655.

12. Brooks A, Schinde V, Bateman AC, et al. Interstitial fibrosis in the dilated non-ischaemic myocardium. Heart. 2003; 89(10): 1255-1256, indexed in Pubmed: 12975439.

13. Chyrchel B, Totoń-Żurańska J, Kruszelnicka O, et al. Association of plasma miR-223 and platelet reactivity in patients with coronary artery disease on dual antiplatelet therapy: A preliminary report. Platelets. 2015; 26(6): 593-597, doi: 10.3109/09537104.2014.974527, indexed in Pubmed: 25350775.
14. Rubiś P, Wiśniowska-Śmialek S, Wypasek E, et al. Fibrosis of extracellular matrix is related to the duration of the disease but is unrelated to the dynamics of collagen metabolism in dilated cardiomyopathy. Inflamm Res. 2016; 65(12): 941-949, doi: 10.1007/s00011-016-0977-3, indexed in Pubmed: 27516211.

15. Rouet-Benzineb P, Buhler JM, Dreyfus P, et al. Altered balance between matrix gelatinases (MMP-2 and MMP-9) and their tissue inhibitors in human dilated cardiomyopathy: potential role of MMP-9 in myosin-heavy chain degradation. Eur J Heart Fail. 1999; 1(4): 337-352, indexed in Pubmed:10937947.

16. Herpel E, Singer S, Flechtenmacher C, et al. Extracellular matrix proteins and matrix metalloproteinases differ between various right and left ventricular sites in end-stage cardiomyopathies. Virchows Arch. 2005; 446(4): 369-378, doi: 10.1007/s00428-004-1177-z, indexed in Pubmed:15806380.

17. Tyagi SC, Campbell SE, Reddy HK, et al. Matrix metalloproteinase activity expression in infarcted, noninfarcted and dilated cardiomyopathic human hearts. Mol Cell Biochem. 1996; 155(1): 13-21, indexed in Pubmed: 8717434.

18. Küçüker SA, Stetson SJ, Becker KA, et al. Evidence of improved right ventricular structure after LVAD support in patients with end-stage cardiomyopathy. J Heart Lung Transplant. 2004; 23(1): 28-35, indexed in Pubmed: 14734124.

19. Kakimoto Yu, Tanaka M, Kamiguchi H, et al. MicroRNA deep sequencing reveals chamber-specific miR-208 family expression patterns in the human heart. Int J Cardiol. 2016; 211: 43-48, doi: 10.1016/j.ijcard.2016.02.145, indexed in Pubmed: 26974694.

20. Zhang $\mathrm{Y}$, Wang $\mathrm{X}, \mathrm{Xu} \mathrm{X}$, et al. Distinct microRNA expression signatures in human right atrial and ventricular myocardium. Mol Cell Biochem. 2012; 371(1-2): 23-29, doi: 10.1007/s11010-012-1417-5, indexed in Pubmed: 22890914.

21. De Rosa S, Fichtlscherer S, Lehmann R, et al. Transcoronary concentration gradients of circulating microRNAs. Circulation. 2011; 124(18): 1936-1944, doi: 10.1161/CIRCULATIONAHA.111.037572, indexed in Pubmed: 21969012.

22. Melman YF, Shah R, Danielson K, et al. Circulating microRMA-30d is associated with response to cardiac resynchronization therapy in heart failure and regulates cardiomyocyte apoptosis: a translational pilot study. Circulation. 2015; 131(25): 2202-2216, doi:10.1161/CIRCULATIONAHA.114.013220, indexed in Pubmed: 25995320.

23. Li D, Ji L, Liu L, et al. Characterization of circulating microRNA expression in patients with a ventricular septal defect. PLoS One. 2014; 9(8): e106318, doi: 10.1371/journal.pone.0106318, indexed in Pubmed: 25165856.

24. Sucharov CC, Sucharov J, Karimpour-Fard A, et al. Micro-RNA expression in hypoplastic left heart syndrome. J Card Fail. 2015; 21(1): 83-88, doi:10.1016/j.cardfail.2014.09.013, indexed in Pubmed: 25291457.

25. Lai CTM, Ng EKO, Chow Pc, et al. Circulating microRNA expression profile and systemic right ventricular function in adults after atrial switch operation for complete transposition of the great arteries. BMC Cardiovasc Disord. 2013; 13: 73, doi: 10.1186/1471-2261-13-73, indexed in Pubmed: 24040857.

26. Tutarel O, Dangwal S, Bretthauer J, et al. Circulating miR-423_5p fails as a biomarker for systemic ventricular function in adults after atrial repair for transposition of the great arteries. Int J Cardiol. 2013; 167(1): 63-66, doi: 10.1016/j.ijcard.2011.11.082, indexed in Pubmed: 22188991.

27. Satoh M, Minami Y, Takahashi Y, et al. Expression of microRNA-208 is associated with adverse clinical outcomes in human dilated cardiomyopathy. J Card Fail. 2010; 16(5): 404-410, doi: 10.1016/j.cardfail.2010.01.002, indexed in Pubmed: 20447577.

28. Devaux Y, Vausort M, McCann GP, et al. A panel of 4 microRNAs facilitates the prediction of left ventricular contractility after acute myocardial infarction. PLoS One. 2013; 8(8): e70644, doi: 10.1371/ journal.pone.0070644, indexed in Pubmed: 23967079.

29. Wang $\mathrm{H}$, Chen F, Tong J, et al. Circulating microRNAs as novel biomarkers for dilated cardiomyopathy. Cardiol J. 2017; 24(1): 65-73, doi:10.5603/CJ.a2016.0097, indexed in Pubmed: 27748501. 